\title{
Comprehensive Benefit Allocation for Joint Operation of Multiple Reservoirs in the Ningbo Based on Shapely-Value Method and Benefit-Incremental Method
}

\author{
Peibing Song ${ }^{1}$, Chao Wang ${ }^{2, *}$, Guanshe Yang ${ }^{3}$, Xiao Jing ${ }^{3}$, Gongbo Ding ${ }^{4}$ \\ ${ }^{1}$ College of Civil Engineering and Architecture, Zhejiang University, Hangzhou 310058, China; songpeibing@zju.edu.cn \\ ${ }^{2}$ State Key Laboratory of Simulation and Regulation of Water Cycle in River Basin, China Institute of Water Resources and \\ Hydropower Research, Beijing 100038, China; wangchao@iwhr.com \\ ${ }^{3}$ Ningbo Raw Water Group Co., Ltd., Ningbo 315000, China; 121938893@qq.com; 1032575829@qq.com \\ ${ }^{4}$ School of Water Resources and Environment, China University of Geosciences (Beijing), Beijing, 100083, China; 625613932@qq.com
}

\begin{abstract}
With the completion of interconnection project of the Eastern Reservoir Group, it is possible to realize the joint operation of the multiple reservoirs in the Ningbo, and integrated dispatching involving water supply and power generation has significant benefits. Based on the inequality of multi-stakeholders and the irrationality of benefit allocation in the cascade reservoir, this paper takes the joint operation of three reservoirs in Ningbo as an example and establishes a Shapley-value and benefit-incremental model, aiming at quantifying and sharing the compensation benefits. Results show that this method not only has strong operability, but also it has clear objectives that can be quantified. Moreover, this method takes the actual contribution value of different stakeholders into consideration. Thus, adopting this method is able to give full play to the enthusiasm of different reservoirs and achieve the maximum comprehensive benefit of the reservoir group.
\end{abstract}

\section{Introduction}

Urban water resources refer to all natural water resources and renewable water resources that can be used by cities. It is the basis for the formation and development of cities and the source of urban water supply ${ }^{[1]}$. Characteristics of urban water resources in China are low per capita occupancy volume, severely uneven spatial-temporal distribution, and high intensity of development and utilization [2]. Moreover, with the acceleration of urbanization and the growth of urban population, problems of urban water problems are more prominent. In recent years, frequent urban water pollution accidents, single water-supply source, and lack of emergency water source have seriously affected social social stability and sustainable economic development. Therefore, exploring the potential of local water resources and carrying out the joint operation of multi-sources can effectively alleviate the contradiction between water supply and water demand of urban water resources ${ }^{[3]}$.

Urban reservoir is a comprehensive reservoir that is mainly for basin flood control and urban water supply, which takes power generation and agricultural irrigation into consideration at the same time ${ }^{[4]}$. In the actual joint operation of multiple reservoirs, not only the rational allocation of water quantity should be considered, but also the economic benefits should should be brought into full play ${ }^{[5]}$. However, problems such as unique stakeholders and unreasonable benefit allocation will extremely influence the motivation of multi-stakeholders, which is not conductive to the cascade operation and the improvement of overall comprehensive benefits ${ }^{[5,6]}$. In this paper, the shapely-value method and benefitincremental method are adopted to solve the problem of comprehensive benefit allocation of multiple reservoirs, which is applied to quantify and allocate compensation benefits.

\section{Research method}

\subsection{Shapely-value method}

Shapely L.S. proposed the shapely-value method in 1953, and this method is a mathematical method for solving the problem of benefit allocation involving multistakeholders, which can obtain the unique solution in the cooperative game ${ }^{[7-9]}$. When the cooperative benefits generated from two or more decision-makers participating in the cooperation are greater than the sum of their respective independent action, each participant hopes to obtain a satisfactory proportion from the cooperative benefit ${ }^{[8,9]}$. According to the shapely-value method, each participant's payoff equals a weighted average contribution of participants to every coalition ${ }^{[10]}$. Shapely L.S thought the payoff to the $i$ th participant as follows:

*Correspondence: wangchao@iwhr.com; Tel.: +86-173-1919-6286 


$$
\left\{\begin{array}{l}
\varphi_{i}(v)=\sum_{s \in S_{i}} \omega(|s|)[v(s)-v(s \backslash\{i\})] \\
\omega(|s|)=\frac{(|s|-1) !(n-|s|) !}{n !}
\end{array}\right.
$$

Where, $S_{i}$ is the set formed by all subsets containing of $i$ th participant; $|s|$ is the number of $s$ element in the set; $\omega(|s|)$ is the weight factor; $v(s)-v(s \backslash\{i\})$ is the contribution made by participant $i$ in its coalition $s$.

When the coalition is made up of two participants, the shapely-value benefit allocation is calculated as follow:

$$
\left\{\begin{array}{l}
\phi_{1}(l)=[l(m)+l(m n)-l(n)] / 2 \\
\phi_{2}(l)=[l(n)+l(m n)-l(m)] / 2
\end{array}\right.
$$

Where, $\phi_{1}(l)$ and $\phi_{2}(l)$ are the actual benefits of two participants obtained from the cooperative benefit according to the shapely-value method, respectively; $l(m)$ and $l(n)$ are the actual benefit when two participants operate separately. $l(m n)$ is the total benefit of the one participant cooperating with the other one.

When the coalition is made up of three participants, the shapely-value benefit allocation is calculated as follow: $\left\{\begin{array}{l}\varphi_{1}(v)=[2 v(a)+v(a b)-v(b)+v(a c)-v(c)+2 v(a b c)-2 v(b c)] / 6 \\ \varphi_{2}(v)=[2 v(b)+v(a b)-v(c)+v(b c)-v(a)+2 v(a b c)-2 v(a c)] / 6 \\ \varphi_{3}(v)=[2 v(c)+v(a c)-v(a)+v(b c)-v(b)+2 v(a b c)-2 v(a b)] / 6\end{array}\right.$

Where, $\varphi_{1}(v), \varphi_{2}(v)$ and $\varphi_{3}(v)$ are the actual benefits of the first, second, and third participants obtained from the cooperative benefit according to the shapely-value method, respectively; $v(a), v(b)$ and $v(c)$ are the actual benefit when the first, second, and third participants operate separately. $v(a b)$ is the total benefit of the first participant cooperating with the second participant; $v(a c)$ is the total benefit of the first participant cooperating with the third participant; $v(b c)$ is the total benefit of the second participant cooperating with the third participant; $v(a b c)$ is the total benefit of the first participant and the second participant cooperating with the third participant.

\subsection{Benefit-incremental method}

The comprehensive benefit of the urban reservoir consist of water supply benefit and power generation benefit, when the reservoir operates separately:

$$
\left\{\begin{array}{l}
B_{c, i}=B_{w, i}+B_{g, i} \\
B_{c}=\sum_{i=1}^{n} B_{c, i}
\end{array}\right.
$$

Where, $B_{c, i}, B_{w, i}$ and $B_{g, i}$ are the comprehensive benefit, water supply benefit and power generation benefit of the $i$ th reservoir when it is dispatched separately, respectively; $B_{c}$ is the total comprehensive benefit of the $n$ reservoirs when they are dispatched separately. When the reservoir operates jointly:

$$
\left\{\begin{array}{l}
B_{c, i}^{\prime}=B_{w, i}^{\prime}+B_{g, i}^{\prime} \\
B_{c}^{\prime}=\sum_{i=1}^{n} B_{c, i}^{\prime}
\end{array}\right.
$$

Where, $B_{c, i}^{\prime}, B_{w, i}^{\prime}$ and $B_{g, i}^{\prime}$ are the comprehensive benefit, water supply benefit and power generation benefit of the $i$ th reservoir when it is dispatched jointly, respectively; $B_{c}^{\prime}$ is the total comprehensive benefit of the $n$ reservoirs when they are dispatched jointly.

Then, the total comprehensive benefit increment of the reservoirs and the comprehensive benefit increment of the $i$ th reservoir are calculated as follows:

$$
\left\{\begin{array}{l}
\Delta B_{c}=B_{c}^{\prime}-B_{c}=\sum_{i=1}^{n} B_{c, i}^{\prime}-\sum_{i=1}^{n} B_{c, i} \\
\Delta B_{c, i}=\Delta B_{c} \times \frac{B_{c, i}}{B_{c}}
\end{array}\right.
$$

Therefore, the distributed comprehensive benefit of the $i$ th reservoir according to the benefit-incremental method is calculated as follows:

$$
B_{c, i}^{\prime \prime}=B_{c, i}+\Delta B_{c, i}=B_{c, i}+\left(B_{c}^{\prime}-B_{c}\right) \times \frac{B_{c, i}}{B_{c}}=B_{c, i}+\left(\sum_{i=1}^{n} B_{c, i}^{\prime}-\sum_{i=1}^{n} B_{c, i}\right) \times \frac{B_{c, i}}{B_{c}}
$$

\section{Case study}

With the completion of interconnection project of the Eastern Reservoir Group, it is possible to realize the joint operation of the multiple reservoirs in the Ningbo. Aiming at the problem that multiple reservoirs belong to the different stakeholders, three reservoirs in the Ningbo was taken as an example in this paper, and the shapely-value method and the benefit-incremental method are applied for the compensation benefit generated from the joint operation of multiple reservoirs ${ }^{[10]}$. Results show that these methods take the actual contribution of different stakeholders into account and the objective function of the maximum comprehensive benefit in the joint operation of multiple reservoirs can be achieved.

\subsection{General introduction}

This dispatch system consists of three reservoirs, namely the Baixi reservoir, the Xixi reservoir and the Huangtan reservoir. Among them, the Baixi reservoir is a part of Ningbo Raw Water Group Co., Ltd., while the Xixi reservoir and the Huangtan reservoir are belong to Ninghai County Water Conservancy Bureau. The basic characteristic parameters are shown in Table 1, and the topological relation of the three reservoirs based on the geographical location is shown in Figure 1.

According to the Figure 1, the Xixi-Huangtan cascade reservoir, composed of the Xixi reservoir and the Huangtan reservoir, is combined with the Baixi reservoir to provide raw water to the downstream water plant. When 
the three reservoirs are dispatched separately, the daily water supply of the Baixi reservoir is five hundred thousand tons/day, and that of the Xixi-Huangtan cascade reservoir is one hundred thousand tons/day. When the three reservoirs are dispatched jointly, the daily water supply of the Baixi-Xixi-Huangtan cascade reservoir is six hundred thousand tons/day. In addition, the water supply source of the water plant can take water from the downstream of the Baixi reservoir or the upstream of the Huangtan reservoir.

Table 1 Characteristic parameters of three reservoirs located in the Eastern Reservoir Group

\begin{tabular}{cccc}
\hline Reservoir & Baixi & Xixi & Huangtan \\
Normal water level (m) & 170 & 147 & 78.8 \\
Water supply dead water level (m) & 140 & 117 & 54.75 \\
Power generation dead water level (m) & 100 & 100 & 50 \\
Tail water level (m) & 80 & 84.5 & 960 \\
Installed capacity $(\mathrm{kw})$ & 18000 & 6000 & 0.5 \\
Water price $(\mathrm{yuan} / \mathrm{ton})$ & 0.5 & 0.5 & 0.4 \\
Electricity price $(\mathrm{yuan} / \mathrm{kW} \cdot \mathrm{h})$ & 0.4 & 0.4 & 0.4 \\
\hline
\end{tabular}

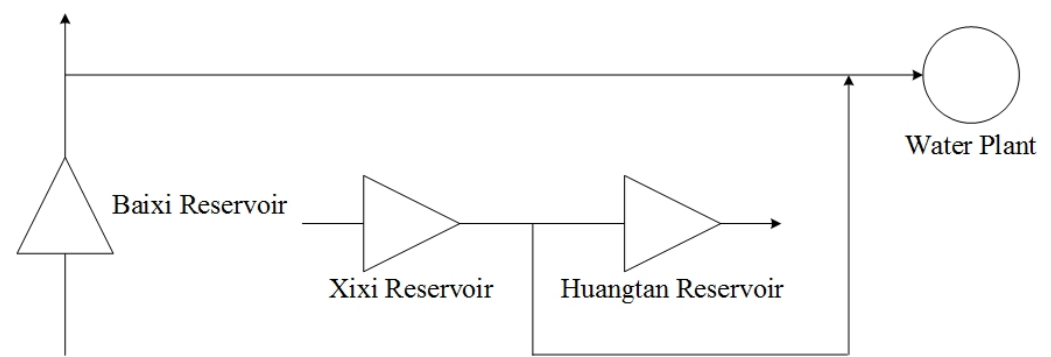

Figure 1 Schematic map of three reservoirs and one water plant in the study area

\subsection{Scheduling model}

The Baixi reservoir and the Xixi-Huangtan cascade reservoir are important parts that the Eastern Reservoir Group of Ningbo supply water to the city, and its comprehensive benefit is mainly reflected in two respects, namely, water supply and power generation. In this paper, a mathematical model is established based on meeting water demand of the water plant, taking the maximum comprehensive benefit as the objective function.

\subsubsection{Objective function}

For the reservoir group with the urban water supply as one of its main objectives, the maximum comprehensive benefit model is premised on ensuring the daily water demand of the water plant in priority, and then repeatedly adjusts the daily water supply of each reservoir and determines the power generation process of different reservoirs. Therefore, the maximum of reservoir group can be achieved and its objective function:

$$
O B J=\max \sum_{i=1}^{3} B_{c, i}=\max \sum_{i=1}^{3}\left(B_{w, i}+B_{g, i}\right)
$$

Where, $i=1, i=2$ and $i=3$ stand for the Baixi reservoir, the Xixi reservoir and the Huangtan reservoir, respectively. Water supply benefit and power generation benefit of the $i$ th reservoir can be calculated by the following formula:

$\left\{\begin{array}{l}B_{w, i}=\left(Q_{w, i} \times T_{w} \times 24 \times 3600 \times P_{w}\right) / 10000 \\ B_{g, i}=\left(P_{g, i} \times T_{g} \times 24 \times P_{g}\right) / 10000\end{array} \quad i=1,2,3\right.$

Where, $Q_{w, i}, P_{g, i}$ are the water supply and the power generation flow of the $i$ th reservoir, respectively. $T_{w}$ and $T_{g}$ are the time length of the water supply and the power generation, respectively. $P_{w}$ and $P_{g}$ are the water price and the electricity price, respectively.

\subsubsection{Constraint conditions}

When the water supply and power generation of the reservoir conflict, the water balance formula is:

$$
V_{i, j+1}=V_{i, j}+3600\left(I_{i, j}-G_{i, j}-W_{i, j}-S_{i, j}\right) \times \Delta T_{j}
$$


When the water supply and power generation of the reservoir do not conflict, the water balance formula is:

$$
\left\{\begin{array}{l}
V_{i, j+1}=V_{i, j}+3600\left(I_{i, j}-Q_{i, j}-S_{i, j}\right) \times \Delta T_{j} \\
Q_{i, j}=\max \left\{G_{i, j}, W_{i, j}\right\}
\end{array}\right.
$$

Where, $V_{i, j}$ is the storage capacity of the $i$ th reservoir in the $j$ th time period. $I_{i, j}, G_{i, j}, W_{i, j}, S_{i, j}$ and $Q_{i, j}$ are the inflow, power generation flow, water supply flow, surplus water and outflow, respectively.

Apart from initial conditions such as the initial water level, the end water level, the monthly runoff process and the time length of three reservoirs, other constraint conditions such as the water level constraints, the flow constraints and the output constraints are also considered.

\section{Benefit analysis}

The calculation is executed according to the different operation modes, and the calculation results are shown in Table 2.

According to the Table 2, when the three reservoirs are dispatched separately, the comprehensive benefit generated by the Baixi reservoir is 104.82 billion yuan, and that of the Xixi-Huangtan cascade reservoir is 28.16 million yuan. At this time, the total comprehensive benefit generated by the reservoir group is 132.98 million yuan. When the three reservoirs are dispatched jointly, the comprehensive benefit generated by the Baixi reservoir is 118.12 million yuan, and that of the Xixi-Huangtan cascade reservoir is 20.18 million yuan. At this time, the total comprehensive benefit generated by the reservoir group is 138.3 million yuan.

Table 2 Benefits of reservoir operation under different operation modes

\begin{tabular}{ccccc}
\hline \multirow{2}{*}{ Operation mode } & \multicolumn{2}{c}{ Separated operation } & \multicolumn{2}{c}{ Joint operation } \\
\cline { 2 - 5 } & Baixi & $\begin{array}{c}\text { Xixi- } \\
\text { Huangtan }\end{array}$ & Baixi & $\begin{array}{c}\text { Xixi- } \\
\text { Huangta } \\
\text { n }\end{array}$ \\
\hline $\begin{array}{c}\text { Water supply benefit (million yuan) } \\
\text { Power generation benefit (million } \\
\text { yuan) }\end{array}$ & 83.21 & 18.24 & 96.77 & 9.62 \\
\hline $\begin{array}{c}\text { Comprehensive benefit (million } \\
\text { yuan) }\end{array}$ & $\begin{array}{c}104.8 \\
2\end{array}$ & 21.6 & 21.35 & 10.56 \\
\hline
\end{tabular}

\subsection{Shapely-value method}

The shapley-value method is adopted to calculate the actual comprehensive benefit of the three reservoirs with reference to the formula (2).

$\left\{\begin{array}{l}\phi(B a i)=\frac{l(\text { Bai })+l(\text { Bai }-X i-\text { Huang })-l(X i-\text { Huang })}{2}=107.48 \\ \phi_{2}(X i-\text { Huang })=\frac{l(X i-\text { Huang })+l(\text { Bai }-X i-\text { Huang })-l(\text { Bai })}{2}=30.82\end{array}\right.$

Where, $\quad l(B a i) \quad, \quad l(X i-H u a n g) \quad$ and $l(B a i-X i-H u a n g)$ are the comprehensive benefit of the Baixi reservoir, the Xixi-Huangtan cascade reservoir and the Baixi-Xixi-Huangtan cascade reservoir. $\phi_{1}(\mathrm{Bai})$ and $\phi_{2}(X i-H u a n g)$ are the actual comprehensive benefit of the Baixi reservoir and the Xixi-Huangtan cascade reservoir.

\subsection{Benefit-incremental method}

The comprehensive benefit of the Xixi reservoir, the Huangtan reservoir and the Xixi-Huangtan reservoir under different operation modes are shown in Table 3.

Table 3 The comprehensive benefit of the Xixi reservoir, the Huangtan reservoir and the Xixi-Huangtan reservoir under different operation modes

\begin{tabular}{|c|c|c|c|c|}
\hline \multirow[b]{2}{*}{ operation mode } & \multicolumn{3}{|c|}{ separated operation } & $\begin{array}{l}\text { Shapley-value } \\
\text { method }\end{array}$ \\
\hline & Xixi & $\begin{array}{c}\text { Huang } \\
\tan \end{array}$ & $\begin{array}{c}\text { Xixi- } \\
\text { Huangta } \\
n\end{array}$ & Xixi-Huangtan \\
\hline $\begin{array}{l}\text { comprehensive benefit } \\
\text { (million yuan) }\end{array}$ & 6.45 & 21.71 & 28.16 & 30.82 \\
\hline
\end{tabular}

Then, the Benefit-incremental method is adopted to calculate the distributed comprehensive benefit of the Xixi reservoir and the Huangtan reservoir according to the formula (7), and the calculation results are shown as follows:

$\left\{\begin{array}{l}B_{c, x i}^{\prime \prime}=7.06 \\ B_{c, \text { huang }}^{\prime \prime}=23.76\end{array}\right.$

Table 4 The comprehensive benefit of the three reservoirs under different operation modes

\begin{tabular}{cccc}
\hline Operation mode & Baixi & Xixi & Huangtan \\
\hline Separated operation (million yuan) & 104.82 & 6.45 & 21.71 \\
\hline Joint operation (million yuan) & 118.12 & 6.45 & 13.73 \\
\hline Shapely-value method and benefit-incremental method (million yuan) & 107.48 & 7.06 & 23.76 \\
\hline Comprehensive benefit increment (million yuan) & 2.66 & 0.61 & 2.05 \\
\hline
\end{tabular}

The comprehensive benefit of the Xixi reservoir and the Huangtan reservoir under the separated operation mode are 6.45 million yuan and 21.71 million yuan, respectively. While the comprehensive benefit of the three reservoirs under different operation modes are shown in Table 4.

According to the Table 4, compared to the separated operation, the comprehensive benefit increment of the three reservoirs based on the shapely-value method and the benefit-incremental method are 2.66 million yuan, 0.61 million yuan and 2.05 million yuan, respectively.

\section{Conclusion}

(1) The benefit allocation model based on the shapelyvalue method and the benefit-incremental method can quantify and share the compensation benefits. Moreover, the sharing results reasonable and stable.

(2) The joint operation model proposed in this paper considers the water supply benefit and the power generation benefit of each reservoir, which is conductive to achieving the maximum comprehensive benefit of the cascade reservoir.

\section{Acknowledgements}

This research was financially supported by the National 
Natural Science Foundation of China (51709275) and the Zhejiang Water Conservancy Science and Technology Project (RA1703).

\section{References:}

1. Cheng H, Hu Y A, Zhao J F. Meeting China's water shortage crisis: current practices and challenges[J]. Environmental Science \& Technology, 2009,43(2):240-244.

2. Zhang H, Jin G, Yu Y. Review of River Basin Water Resource Management in China[J]. Water, 2018,10(4):425.

3. Oliveira R, Loucks D P. Operating rules for multireservoir systems[J]. Water Resources Research, 1997,33(4):839-852.

4. Perera B J C, Codner G P. Reservoir Targets for Urban Water Supply Systems[J]. Journal of Water Resources Planning \& Management, 1996,122(4):270-279.

5. Wang L Z. Water Resources Allocation: A Cooperative Game Theoretic Approach[J]. Journal of Environmental Informatics, 2003,2(2):11-22.

6. Teasley R L, McKinney D C. Calculating the Benefits of Transboundary River Basin Cooperation: Syr Darya Basin[J]. Journal of Water Resources Planning and Management, 2011,137(6):481-490.

7. Smith J M. Evolution and the Theory of Games[M]. Cambridge University Press, 1982, 41-45.

8. Chun Y. A new axiomatization of the shapley value[J]. Games and Economic Behavior, 1989,1(2):119-130.

9. Fatima S S, Wooldridge M, Jennings N R. A linear approximation method for the Shapley value[J]. Artificial Intelligence, 2008,172(14):1673-1699.

10. Jafarzadegan K, Abed-Elmdoust A, Kerachian R. A stochastic model for optimal operation of inter-basin water allocation systems: a case study[J]. Stochastic Environmental Research and Risk Assessment, 2014,28(6):1343-1358. 